\title{
Logical entropy of dynamical systems in product MV-algebras and general scheme
}

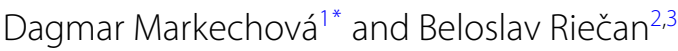

"Correspondence:

dmarkechova@ukf.sk

${ }^{1}$ Department of Mathematics,

Faculty of Natural Sciences,

Constantine the Philosopher University in Nitra, Nitra, Slovakia

Full list of author information is

available at the end of the article

\begin{abstract}
The present paper is aimed at studying the entropy of dynamical systems in product MV-algebras. First, by using the concept of logical entropy of a partition in a product MV-algebra introduced and studied by Markechová et al. (Entropy 20:129, 2018), we define the logical entropy of a dynamical system in the studied algebraic structure. In addition, we introduce a general type of entropy of a product MV-algebra dynamical system that includes the logical entropy and the Kolmogorov-Sinai entropy as special cases. It is proved that the proposed entropy measure is invariant under isomorphism of product MV-algebra dynamical systems.
\end{abstract}

MSC: 28AXX; 37A35; 54C70

Keywords: Product MV-algebra; Partition; Sub-additive generator; Entropy; Dynamical system

\section{Introduction}

The Shannon entropy [2] is the basic notion of information theory (cf. [3]). If an experiment has $n$ results with probabilities $p_{1}, p_{2}, \ldots, p_{n}$, then its entropy is the sum $\sum_{i=1}^{n} s\left(p_{i}\right)$, where $s:[0,1] \rightarrow[0, \infty)$ is Shannon's entropy function defined by equation

$$
s(x)=-x \log x
$$

for every $x \in[0,1](0 \log 0$ is defined to be 0$)$. Many years later, the Shannon entropy was used surprisingly in a quite different area of theory as well as in practice, i.e., in dynamical systems. Recall that a classical dynamical system is a quaternion $(\Omega, S, P, T)$, where $(\Omega, S, P)$ is a probability space and $T: \Omega \rightarrow \Omega$ is a measure preserving map, i.e., $P\left(T^{-1}(B)\right)=P(B), B \in S$. If $\mathcal{B}=\left\{B_{1}, B_{2}, \ldots, B_{n}\right\}$ is a measurable partition of $\Omega$ with probabilities $p_{1}, p_{2}, \ldots, p_{n}$ of the corresponding elements, then its entropy is again $H(\mathcal{B})=$ $\sum_{i=1}^{n} s\left(p_{i}\right)=-\sum_{i=1}^{n} p_{i} \cdot \log p_{i}$. If $\mathcal{B}=\left\{B_{1}, B_{2}, \ldots, B_{n}\right\}$ and $\mathcal{C}=\left\{C_{1}, C_{2}, \ldots, C_{m}\right\}$ are measurable partitions of $\Omega$, then the measurable partition $\mathcal{B} \vee \mathcal{C}=\left\{B_{i} \cap C_{j} ; i=1,2, \ldots, n, j=1,2, \ldots, m\right\}$ represents an experiment consisting of a realization of experiments $\mathcal{B}$ and $\mathcal{C}$. Further, by $T^{-1}(\mathcal{B})$ the measurable partition $\left\{T^{-1}\left(B_{1}\right), T^{-1}\left(B_{2}\right), \ldots, T^{-1}\left(B_{n}\right)\right\}$ is denoted. The entropy of a dynamical system $(\Omega, S, P, T)$ has been defined by Kolmogorov and Sinai $[4,5]$ as the number $H(T)=\sup H(\mathcal{B}, T) ; \mathcal{B}$ is a finite measurable partition of $\{\Omega\}$, where $H(\mathcal{B}, T)=$ $\lim _{n \rightarrow \infty} \frac{1}{n} H\left(\bigvee_{i=0}^{n-1} T^{-i}(\mathcal{B})\right)$. It is used to measure dynamical complexity of the considered

(c) The Author(s) 2019. This article is distributed under the terms of the Creative Commons Attribution 4.0 International License (http://creativecommons.org/licenses/by/4.0/), which permits unrestricted use, distribution, and reproduction in any medium, provided you give appropriate credit to the original author(s) and the source, provide a link to the Creative Commons license, and indicate if changes were made. 
dynamical system. The number $H(T)$ is also a useful instrument for distinguishing dynamical systems. Namely, if two dynamical systems are isomorphic, then they have the same entropy. By this way Kolmogorov and Sinai showed that there are non-isomorphic Bernoulli shifts. Recall that the opposite implication holds, but only for Bernoulli shifts: if two Bernoulli shifts have the same entropy, they are isomorphic [6, 7].

The successful using of the Kolmogorov and Sinai entropy of dynamical systems has led to an intensive study of various aspects of alternative entropy measures of dynamical systems. We note that in the recently published paper [8], the notion of logical entropy $H_{l}(T)$ of a dynamical system $(\Omega, S, P, T)$ was proposed and studied. It has been shown that by replacing the Shannon entropy function by the logical entropy function $l:[0,1] \rightarrow$ $[0, \infty)$ defined by

$$
l(x)=x(1-x)
$$

for every $x \in[0,1]$, we get the results that are analogous to the case of classical Kolmogorov-Sinai entropy theory. It has been proven that the logical entropy $H_{l}(T)$ distinguishes non-isomorphic dynamical systems; so it can be used as an alternative instrument for distinguishing them. Note that some other recently published results regarding the logical entropy measure can be found, for example, in [9-17].

Actually, all of the above-mentioned studies are possible in the Kolmogorov probability theory based on the modern integration theory. It gives a possibility to describe and study some problems of uncertainty. Of course, in 1965, Zadeh presented another approach to uncertainty in his article [18]. While the Kolmogorov probability applications are based on objective measurements, the Zadeh fuzzy theory is based on subjective improvements. Of course, one of the first Zadeh articles on the fuzzy set theory was devoted to probability on fuzzy sets (cf. [19]). Therefore, the entropy of fuzzy dynamical systems has also been studied (cf. [20-23]). Recall that the fuzzy set is a mapping $f: \Omega \rightarrow[0,1](f(\omega)$ is interpreted as the degree of the element $\omega \in \Omega$ to the considered fuzzy set $f$ ), hence the fuzzy partition of $\Omega$ is a family of fuzzy sets $A=\left\{f_{1}, f_{2}, \ldots, f_{n}\right\}$ such that $\sum_{i=1}^{n} f_{i}=1$. And again we can meet the Shannon formula: $H(A)=-\sum_{i=1}^{n} p_{i} \log p_{i}$, where $p_{i}=\int_{\Omega} f_{i} d P$ (cf. [23]). An overview of publications devoted to the entropy of fuzzy dynamical systems can be found in [24].

In [25], Atanassov presented a remarkable generalization of fuzzy sets, i.e., intuitionistic fuzzy sets. An intuitionistic fuzzy set is a pair $A=\left(f_{A}, g_{A}\right)$ of fuzzy sets such that $f_{A}+g_{A} \leq$ 1. Here $f_{A}$ is a membership function, $g_{A}$ a non-membership function. If $f$ is a fuzzy set, then the pair $(f, 1-f)$ is an intuitionistic fuzzy set. Also, the probability on families of intuitionistic fuzzy sets has been studied (cf. [26]).

Anyway, the most useful instrument for describing multivalued processes is an MValgebra [27], especially after its Mundici's characterization as an interval in a lattice ordered group (cf. [28]). This algebraic structure is currently being studied by many researchers and it is natural that there are many results also regarding entropy in this structure; we refer, for instance, to $[29,30]$. A probability theory was studied on MV-algebras as well; for a review, see [31]. Of course, in some problems of probability it is necessary to introduce a product on an MV-algebra, an operation outside the corresponding group addition. The operation of a product on an MV-algebra was introduced independently by Riečan [32] from the point of view of probability and by Montagna [33] from the point of 
view of mathematical logic. Also, the approach from the point of view of a general algebra proposed by Jakubík in [34] seems to be interesting; see also [35]. We note that the notion of product MV-algebra generalizes some families of fuzzy sets; an example of product MV-algebra is a full tribe of fuzzy sets (see, e.g., [24]).

A suitable entropy theory of Shannon and Kolmogorov-Sinai type for the product MValgebras has been provided by Petrovičová in [36, 37]. We remark that in our article [38], based on the results of Petrovičová, we introduced the notions of Kullback-Leibler divergence and mutual information of partitions in a product MV-algebra. The logical entropy, the logical divergence, and the logical mutual information of partitions in a product MValgebra were studied in [1]. In the present paper, we extend the study of logical entropy of partitions in product $\mathrm{MV}$-algebras to the case of product MV-algebra dynamical systems. Moreover, we introduce a general type of entropy of a dynamical system in a product MValgebra. The proposed definition is based on the concept of the sub-additive generator $\varphi$ introduced by the authors in [39].

The rest of the article is organized as follows. Section 2 contains basic definitions, notations, and some known facts that will be used in the paper. Our results are presented in the succeeding two sections. In Sect. 3, we define and study the logical entropy of a dynamical system in a product MV-algebra and examine its properties. In Sect. 4, a general type of entropy of a dynamical system in a product MV-algebra is introduced. It is proved that the proposed entropy measure is invariant under isomorphism of product MV-algebra dynamical systems. It is shown that the logical entropy and the Kolmogorov-Sinai entropy of a dynamical system in a product MV-algebra can be obtained as special cases of the proposed general scheme. It follows that the isomorphic product MV-algebra dynamical systems have the same logical entropy and the same Kolmogorov-Sinai entropy. We illustrate the results with examples. Finally, the last section provides brief closing remarks.

\section{Basic definitions and related works}

We start by reminding the definitions of basic terms and some of the known results that will be used in the article. We mention some works related to the subject of this article, of course, without claiming completeness.

Several different (but equivalent) axiom systems have been used to define the term of MV-algebra (cf., e.g., [32, 40, 41]). In our article, we apply the definition of MV-algebra in accordance with the definition given by Riečan in [42], which is based on the Mundici representation theorem. Based on Mundici's theorem [28] (see also [43]), MV-algebras can be viewed as intervals of an abelian lattice-ordered group (shortly $l$-group). We remind that by an $l$-group (cf. [44]) we understand a triplet $(G,+, \leq)$, where $(G,+)$ is an abelian group, $(G, \leq)$ is a partially ordered set being a lattice, and $x \leq y \Longrightarrow x+z \leq y+z$.

Definition 2.1 ([42]) An MV-algebra is an algebraic structure $\mathcal{A}=(A, \oplus, *, 0, u)$ satisfying the following conditions:

(i) There exists an $l$-group $(G,+, \leq)$ such that $A=[0, u]=\{x \in G ; 0 \leq x \leq u\}$, where 0 is the neutral element of $(G,+)$ and $u$ is a strong unit of $G$ (i.e., $u \in G$ such that $u>0$ and to every $x \in G$ there exists a positive integer $n$ with the property $x \leq n u$ );

(ii) $\oplus, *$ are binary operations on $A$ satisfying the following identities: $x \oplus y=(x+y) \wedge u, x * y=(x+y-u) \vee 0$. 
Definition 2.2 ([31]) A state on an MV-algebra $\mathcal{A}=(A, \oplus, *, 0, u)$ is a mapping $\mu: A \rightarrow$ $[0,1]$ with the following two properties:

(i) $\mu(u)=1$;

(ii) If $x, y \in A$ such that $x+y \leq u$, then $\mu(x+y)=\mu(x)+\mu(y)$.

Definition 2.3 ([42]) A product MV-algebra is an algebraic structure $(A, \oplus, *, \cdot, 0, u)$, where $(A, \oplus, *, 0, u)$ is an MV-algebra and · is an associative and abelian binary operation on $A$ with the following properties:

(i) For every $x \in A, u \cdot x=x$;

(ii) If $x, y, z \in A$ such that $x+y \leq u$, then $z \cdot x+z \cdot y \leq u$, and $z \cdot(x+y)=z \cdot x+z \cdot y$.

For brevity, we will write $(A, \cdot)$ instead of $(A, \oplus, *, \cdot, 0, u)$. A relevant probability theory for the product MV-algebras was developed by Riečan in [45], see also [46, 47]; the entropy theory of Shannon and Kolmogorov-Sinai type for the product MV-algebras was proposed in $[36,37]$. The logical entropy of a partition in a product MV-algebra $(A, \cdot)$ was defined and studied in [1]. We present the main idea and some results of these theories that will be used in the following text.

By a partition in a product MV-algebra $(A, \cdot)$, we understand any $n$-tuple $X=\left(x_{1}, x_{2}, \ldots\right.$, $x_{n}$ ) of elements of $A$ with the property $x_{1}+x_{2}+\cdots+x_{n}=u$. In the system of all partitions in a given product MV-algebra $(A, \cdot)$, we define the refinement partial order $\succ$ in a standard way (cf. [1]). If $X=\left(x_{1}, x_{2}, \ldots, x_{n}\right)$ and $Y=\left(y_{1}, y_{2}, \ldots, y_{m}\right)$ are two partitions in $(A, \cdot)$, then we write $Y \succ X$ (and we say that $Y$ is a refinement of $X$ ), if there exists a partition $\{I(1), I(2), \ldots, I(n)\}$ of the set $\{1,2, \ldots, m\}$ such that $x_{i}=\sum_{j \in I(i)} y_{j}$, for $i=1,2, \ldots, n$. Further, we put $X \vee Y=\left(x_{i} \cdot y_{j} ; i=1,2, \ldots, n, j=1,2, \ldots, m\right)$. Since $\sum_{i=1}^{n} \sum_{j=1}^{m} x_{i} \cdot y_{j}=\left(\sum_{i=1}^{n} x_{i}\right)$. $\left(\sum_{j=1}^{m} y_{j}\right)=u \cdot u=u$, the system $X \vee Y$ is a partition in $(A, \cdot)$; it represents an experiment consisting of a realization of $X$ and $Y$.

Later we shall need the following assertions:

Proposition 2.1 Let $X=\left(x_{1}, x_{2}, \ldots, x_{n}\right)$ be a partition in a product $M V$-algebra $(A, \cdot)$ and $\mu: A \rightarrow[0,1]$ be a state. Then, for any element $y \in A$, it holds $\mu(y)=\sum_{i=1}^{n} \mu\left(x_{i} \cdot y\right)$.

Proof The proof can be found in [1].

Proposition 2.2 If $X, Y, Z$ are partitions in a product $M V$-algebra $(A, \cdot)$, then it holds $X \vee$ $Y \succ X$, and $Y \succ X$ implies $Y \vee Z \succ X \vee Z$.

Proof The proof can be found in [1].

Proposition 2.3 Let $X, Y, V, Z$ be partitions in a product $M V$-algebra $(A, \cdot)$ such that $Y \succ$ $X$ and $Z \succ V$. Then $Y \vee Z \succ X \vee V$.

Proof Assume that $X=\left(x_{1}, x_{2}, \ldots, x_{n}\right), Y=\left(y_{1}, y_{2}, \ldots, y_{m}\right), V=\left(v_{1}, v_{2}, \ldots, v_{p}\right), Z=\left(z_{1}, z_{2}, \ldots\right.$, $\left.z_{q}\right), Y \succ X, Z \succ V$. Then there exists a partition $\{I(1), I(2), \ldots, I(n)\}$ of the set $\{1,2, \ldots, m\}$ such that $x_{i}=\sum_{j \in I(i)} y_{j}$ for $i=1,2, \ldots, n$, and there exists a partition $\{J(1), J(2), \ldots, J(p)\}$ of the set $\{1,2, \ldots, q\}$ such that $v_{r}=\sum_{k \in J(r)} z_{k}$ for $r=1,2, \ldots, p$. Put $I(i, r)=\{(j, k) ; j \in I(i), k \in$ 
$J(r)\}$ for $i=1,2, \ldots, n, r=1,2, \ldots, p$. We get

$$
x_{i} \cdot v_{r}=\left(\sum_{j \in I(i)} y_{j}\right) \cdot\left(\sum_{k \in J(r)} z_{k}\right)=\sum_{(j, k) \in I(i, r)} y_{j} \cdot z_{k}
$$

for $i=1,2, \ldots, n, r=1,2, \ldots, p$, which means that $Y \vee Z \succ X \vee V$.

Definition 2.4 Let $\mu$ be a state on a product MV-algebra $(A, \cdot)$. We say that partitions $X, Y$ in $(A, \cdot)$ are statistically independent with respect to $\mu$ if $\mu(x \cdot y)=\mu(x) \cdot \mu(y)$ for every $x \in X$ and $y \in Y$.

The following definition of entropy of Shannon type was introduced in [36].

Definition 2.5 Let $X=\left(x_{1}, x_{2}, \ldots, x_{n}\right)$ be a partition in a product MV-algebra $(A, \cdot)$, and $\mu: A \rightarrow[0,1]$ be a state. Then the entropy of $X$ with respect to $\mu$ is defined by Shannon's formula:

$$
H_{s}^{\mu}(X)=\sum_{i=1}^{n} s\left(\mu\left(x_{i}\right)\right)
$$

where $s:[0,1] \rightarrow[0, \infty)$ is the Shannon entropy function defined by Eq. (1.1). If $X=$ $\left(x_{1}, x_{2}, \ldots, x_{n}\right)$ and $Y=\left(y_{1}, y_{2}, \ldots, y_{m}\right)$ are two partitions in $(A, \cdot)$, then the conditional entropy of $X$ given $Y$ is defined by

$$
H_{s}^{\mu}(X / Y)=-\sum_{i=1}^{n} \sum_{j=1}^{m} \mu\left(x_{i} \cdot y_{j}\right) \cdot \log \frac{\mu\left(x_{i} \cdot y_{j}\right)}{\mu\left(y_{j}\right)}
$$

In Eq. (2.2), it is assumed that $0 \cdot \log \frac{0}{x}=0$ if $x \geq 0$. The entropy and the conditional entropy of partitions in a product MV-algebra satisfy all properties corresponding to the properties of Shannon's entropy of measurable partitions in the classical case; for more details, see [36]. In particular, it holds $H_{s}^{\mu}(X \vee Y) \leq H_{s}^{\mu}(X)+H_{s}^{\mu}(Y)$ for every partition $X, Y$ in $(A, \cdot)$. The equality holds if and only if $X, Y$ are statistically independent partitions with respect to $\mu$. This means that Shannon's entropy of partitions in a product MV-algebra has the property of additivity and also the property of sub-additivity.

The definition of logical entropy of a partition in a product MV-algebra was introduced in [1] as follows.

Definition 2.6 Let $X=\left(x_{1}, x_{2}, \ldots, x_{n}\right)$ be a partition in a product MV-algebra $(A, \cdot)$, and $\mu: A \rightarrow[0,1]$ be a state. Then the logical entropy of $X$ with respect to $\mu$ is defined by

$$
H_{l}^{\mu}(X)=\sum_{i=1}^{n} l\left(\mu\left(x_{i}\right)\right)
$$

where $l:[0,1] \rightarrow[0, \infty)$ is the logical entropy function defined by Eq. (1.2). If $X=$ $\left(x_{1}, x_{2}, \ldots, x_{n}\right)$ and $Y=\left(y_{1}, y_{2}, \ldots, y_{m}\right)$ are two partitions in $(A, \cdot)$, then the conditional logi- 
cal entropy of $X$ given $Y$ is defined by

$$
H_{l}^{\mu}(X / Y)=\sum_{i=1}^{n} \sum_{j=1}^{m} \mu\left(x_{i} \cdot y_{j}\right)\left(\mu\left(y_{j}\right)-\mu\left(x_{i} \cdot y_{j}\right)\right) .
$$

The basic properties of the logical entropy of partitions in a product MV-algebra were derived in [1]. Specifically, this entropy measure has been shown to have the property of sub-additivity, but it does not have the property of additivity. It satisfies the following property: if $X, Y$ are statistically independent partitions in a product $\mathrm{MV}$-algebra $(A, \cdot)$, then:

$$
1-H_{l}^{\mu}(X \vee Y)=\left(1-H_{l}^{\mu}(X)\right) \cdot\left(1-H_{l}^{\mu}(Y)\right)
$$

Moreover, the proposed logical entropy measure has the following properties: (L1) for every partition $X, Y$ in $(A, \cdot)$, it holds $H_{l}^{\mu}(X \vee Y)=H_{l}^{\mu}(X)+H_{l}^{\mu}(Y / X)$; (L2) for every partition $X, Y$ in $(A, \cdot)$ such that $Y \succ X$, it holds $H_{l}^{\mu}(Y) \geq H_{l}^{\mu}(X)$.

\section{The logical entropy of dynamical systems in product MV-algebras}

In this section, we extend the definition of logical entropy of a partition in a product MValgebra to the case of dynamical systems and prove basic properties of this measure of information. The known Kolmogorov-Sinai theorem on generators is a useful instrument to compute the entropy of a dynamical system. In the final part of this section we provide a logical version of this theorem for the studied case of product MV-algebra.

Definition 3.1 ([37]) By a dynamical system in a product MV-algebra $(A, \cdot)$, we understand a system $(A, \mu, U)$, where $\mu: A \rightarrow[0,1]$ is a state, and $U: A \rightarrow A$ is a map such that $U(u)=u$, and, for every $x, y \in A$, the following conditions are satisfied:

(i) if $x+y \leq u$, then $U(x)+U(y) \leq u$ and $U(x+y)=U(x)+U(y)$;

(ii) $U(x \cdot y)=U(x) \cdot U(y)$;

(iii) $\mu(U(x))=\mu(x)$.

Remark 3.1 For the sake of brevity, we say also a product MV-algebra dynamical system instead of a dynamical system in a product MV-algebra.

Example 3.1 Let $(\Omega, S, P, T)$ be a classical dynamical system. Put $A=\left\{\chi_{B} ; B \in S\right\}$, where $\chi_{B}: \Omega \rightarrow\{0,1\}$ is the characteristic function of the set $B \in S$. The family $A$ is closed under the product of characteristic functions, and it is a special case of product MV-algebras. If we define the mapping $\mu: A \rightarrow[0,1]$ by $\mu\left(\chi_{B}\right)=P(B), B \in S$, then $\mu$ is a state on the product MV-algebra $(A, \cdot)$. In addition, let us define the mapping $U: A \rightarrow A$ by the equality $U\left(\chi_{B}\right)=\chi_{B} \circ T=\chi_{T^{-1}(B)}, \chi_{B} \in A$. Then the system $(A, \mu, U)$ is a dynamical system in the considered product MV-algebra $(A, \cdot)$. A measurable partition $\mathcal{B}=\left\{B_{1}, B_{2}, \ldots, B_{n}\right\}$ of $\Omega$ can be considered as a partition in the product MV-algebra $(A, \cdot)$; it suffices to consider $\chi_{B_{i}}$ instead of $B_{i}$.

Example 3.2 Let $(\Omega, S, P, T)$ be a classical dynamical system. Let $A$ be a family of all $S$ measurable functions $f: \Omega \rightarrow[0,1]$,the so-called full tribe of fuzzy sets (cf. [24]). The 
family $A$ is closed also with respect to the natural product of fuzzy sets, and it is an important case of product MV-algebras. If we define the state $\mu: A \rightarrow[0,1]$ by the equality $\mu(f)=\int_{\Omega} f d P$ for any element $f$ of $A$, and the mapping $U: A \rightarrow A$ by the equality $U(f)=f \circ T, f \in A$, then it is easy to verify that the system $(A, \mu, U)$ is a dynamical system in the considered product MV-algebra $(A, \cdot)$. The notion of a partition in the product MV-algebra $(A, \cdot)$ coincides with the notion of a fuzzy partition.

Let $(A, \mu, U)$ be a dynamical system in a product MV-algebra $(A, \cdot)$, and $X=\left(x_{1}, x_{2}, \ldots, x_{n}\right)$ be a partition in $(A, \cdot)$. Put $U(X)=\left(U\left(x_{1}\right), U\left(x_{2}\right), \ldots, U\left(x_{n}\right)\right)$. Since $x_{1}+x_{2}+\cdots+x_{n}=u$, according to Definition 3.1, we have $U\left(x_{1}\right)+U\left(x_{2}\right)+\cdots+U\left(x_{n}\right)=U\left(x_{1}+x_{2}+\cdots+x_{n}\right)=$ $U(u)=u$, which means that $U(X)$ is also a partition in $(A, \cdot)$.

Proposition 3.1 Let $(A, \mu, U)$ be a dynamical system in a product $M V$-algebra $(A, \cdot)$, and $X, Y$ be partitions in $(A, \cdot)$. Then

(i) $U(X \vee Y)=U(X) \vee U(Y)$;

(ii) $Y \succ X$ implies $U(Y) \succ U(X)$.

Proof (i) Suppose that $X=\left(x_{1}, x_{2}, \ldots, x_{n}\right), Y=\left(y_{1}, y_{2}, \ldots, y_{m}\right)$. By condition (ii) from Definition 3.1, we have

$$
\begin{aligned}
U(X) \vee U(Y) & =\left(U\left(x_{1}\right), U\left(x_{2}\right), \ldots, U\left(x_{n}\right)\right) \vee\left(U\left(y_{1}\right), U\left(y_{2}\right), \ldots, U\left(y_{m}\right)\right) \\
& =\left(U\left(x_{i}\right) \cdot U\left(y_{j}\right) ; i=1,2, \ldots, n, j=1,2, \ldots, m\right) \\
& =\left(U\left(x_{i} \cdot y_{j}\right) ; i=1,2, \ldots, n, j=1,2, \ldots, m\right)=U(X \vee Y) .
\end{aligned}
$$

(ii) Suppose that $X=\left(x_{1}, x_{2}, \ldots, x_{n}\right), Y=\left(y_{1}, y_{2}, \ldots, y_{m}\right), Y \succ X$. Then there exists a partition $\{I(1), I(2), \ldots, I(n)\}$ of the set $\{1,2, \ldots, m\}$ such that $x_{i}=\sum_{j \in I(i)} y_{j}$ for $i=1,2, \ldots, n$. Therefore, by condition (i) from Definition 3.1, we have

$$
U\left(x_{i}\right)=U\left(\sum_{j \in I(i)} y_{j}\right)=\sum_{j \in I(i)} U\left(y_{j}\right) \quad \text { for } i=1,2, \ldots, n .
$$

However, this means that $U(Y) \succ U(X)$.

Define $U^{2}=U \circ U$, and put $U^{k}=U \circ U^{k-1}$ for $k=1,2, \ldots$, where $U^{0}$ is the identical mapping. It is obvious that the map $U^{k}: A \rightarrow A$ satisfies the conditions from Definition 3.1. Hence, for any non-negative integer $k$, the system $\left(A, \mu, U^{k}\right)$ is a dynamical system in a product MV-algebra $(A, \cdot)$.

Theorem 3.1 Let $(A, \mu, U)$ be a dynamical system in a product $M V$-algebra $(A, \cdot)$, and $X, Y$ be partitions in $(A, \cdot)$. Then, for any non-negative integer $k$, the following equalities hold:

(i) $H_{l}^{\mu}\left(U^{k}(X)\right)=H_{l}^{\mu}(X)$;

(ii) $H_{l}^{\mu}\left(U^{k}(X) / U^{k}(Y)\right)=H_{l}^{\mu}(X / Y)$.

Proof Suppose that $X=\left(x_{1}, x_{2}, \ldots, x_{n}\right)$ and $Y=\left(y_{1}, y_{2}, \ldots, y_{m}\right)$.

(i) Since for any non-negative integer $k$ and $i=1,2, \ldots, n$, it holds $\mu\left(U^{k}\left(x_{i}\right)\right)=\mu\left(x_{i}\right)$, we obtain

$$
H_{l}^{\mu}\left(U^{k}(X)\right)=\sum_{i=1}^{n} l\left(\mu\left(U^{k}\left(x_{i}\right)\right)\right)=\sum_{i=1}^{n} l\left(\mu\left(x_{i}\right)\right)=H_{l}^{\mu}(X) .
$$


(ii) Based on the same argument, we get

$$
\begin{aligned}
H_{l}^{\mu}\left(U^{k}(X) / U^{k}(Y)\right) & =\sum_{i=1}^{n} \sum_{j=1}^{m} \mu\left(U^{k}\left(x_{i} \cdot y_{j}\right)\right) \cdot\left(\mu\left(U^{k}\left(y_{j}\right)\right)-\mu\left(U^{k}\left(x_{i} \cdot y_{j}\right)\right)\right) \\
& =\sum_{i=1}^{n} \sum_{j=1}^{m} \mu\left(x_{i} \cdot y_{j}\right)\left(\mu\left(y_{j}\right)-\mu\left(x_{i} \cdot y_{j}\right)\right)=H_{l}^{\mu}(X / Y) .
\end{aligned}
$$

Theorem 3.2 Let $(A, \mu, U)$ be a dynamical system in a product $M V$-algebra $(A, \cdot)$, and $X$ be a partition in $(A, \cdot)$. Then, for $n=2,3, \ldots$, the following equality holds:

$$
H_{l}^{\mu}\left(\bigvee_{k=0}^{n-1} U^{k}(X)\right)=H_{l}^{\mu}(X)+\sum_{i=1}^{n-1} H_{l}^{\mu}\left(X / \bigvee_{k=1}^{i} U^{k}(X)\right)
$$

Proof We use proof by mathematical induction on $n$, starting with $n=2$. For $n=2$, the statement holds by property (L1). We suppose that the statement holds for a given integer $n>1$, and we will prove that it is true for $n+1$. By property (i) from the previous theorem, we get

$$
H_{l}^{\mu}\left(\bigvee_{k=1}^{n} U^{k}(X)\right)=H_{l}^{\mu}\left(U\left(\bigvee_{k=0}^{n-1} U^{k}(X)\right)\right)=H_{l}^{\mu}\left(\bigvee_{k=0}^{n-1} U^{k}(X)\right)
$$

Therefore, using (L1) and our inductive hypothesis, we get

$$
\begin{aligned}
H_{l}^{\mu}\left(\bigvee_{k=0}^{n} U^{k}(X)\right) & =H_{l}^{\mu}\left(\left(\bigvee_{k=1}^{n} U^{k}(X)\right) \vee X\right)=H_{l}^{\mu}\left(\bigvee_{k=1}^{n} U^{k}(X)\right)+H_{l}^{\mu}\left(X / \bigvee_{k=1}^{n} U^{k}(X)\right) \\
& =H_{l}^{\mu}\left(\bigvee_{k=0}^{n-1} U^{k}(X)\right)+H_{l}^{\mu}\left(X / \bigvee_{k=1}^{n} U^{k}(X)\right) \\
& =H_{l}^{\mu}(X)+\sum_{i=1}^{n-1} H_{l}^{\mu}\left(X / \bigvee_{k=1}^{i} U^{k}(X)\right)+H_{l}^{\mu}\left(X / \bigvee_{k=1}^{n} U^{k}(X)\right) \\
& =H_{l}^{\mu}(X)+\sum_{i=1}^{n} H_{l}^{\mu}\left(X / \bigvee_{k=1}^{i} U^{k}(X)\right) .
\end{aligned}
$$

In conclusion, the statement holds by the principle of mathematical induction.

In the following, we will define the logical entropy of a dynamical system $(A, \mu, U)$. First, we define the logical entropy of $U$ relative to a partition $X$ in $(A, \cdot)$. Then we remove the dependence on $X$ to get the logical entropy of a dynamical system $(A, \mu, U)$. We will need the following proposition.

Proposition 3.2 Let $(A, \mu, U)$ be a dynamical system in a product $M V$-algebra $(A, \cdot)$. Then, for any partition $\operatorname{Xin}(A, \cdot)$, there exists the following limit:

$$
\lim _{n \rightarrow \infty} \frac{1}{n} H_{l}^{\mu}\left(\bigvee_{k=0}^{n-1} U^{k}(X)\right)
$$


Proof Put $c_{n}=H_{l}^{\mu}\left(\bigvee_{k=0}^{n-1} U^{k}(X)\right)$ for $n=1,2, \ldots$ Then the sequence $\left\{c_{n}\right\}_{n=1}^{\infty}$ is a sequence of non-negative real numbers satisfying the condition $c_{r+s} \leq c_{r}+c_{s}$ for every $r, s \in \mathbb{N}$. Indeed, by means of sub-additivity of logical entropy and property (i) from Theorem 3.1, we can write

$$
\begin{aligned}
c_{r+s} & =H_{l}^{\mu}\left(\bigvee_{k=0}^{r+s-1} U^{k}(X)\right) \leq H_{l}^{\mu}\left(\bigvee_{k=0}^{r-1} U^{k}(X)\right)+H_{l}^{\mu}\left(\bigvee_{k=r}^{r+s-1} U^{k}(X)\right) \\
& =c_{r}+H_{l}^{\mu}\left(U^{r}\left(\bigvee_{k=0}^{s-1} U^{k}(X)\right)\right)=c_{r}+H_{l}^{\mu}\left(\bigvee_{k=0}^{s-1} U^{k}(X)\right)=c_{r}+c_{s} .
\end{aligned}
$$

This property guarantees (in view of Theorem 4.9, [48]) the existence of $\lim _{n \rightarrow \infty} \frac{1}{n} c_{n}$.

Definition 3.2 Let $(A, \mu, U)$ be a dynamical system in a product MV-algebra $(A, \cdot)$, and $X$ be a partition in $(A, \cdot)$. Then we define the logical entropy of $U$ relative to $X$ by

$$
H_{l}^{\mu}(U, X)=\lim _{n \rightarrow \infty} \frac{1}{n} H_{l}^{\mu}\left(\bigvee_{k=0}^{n-1} U^{k}(X)\right) .
$$

Remark 3.2 Consider any dynamical system $(A, \mu, U)$ in a product MV-algebra $(A, \cdot)$. If we put $E=(u)$, then $E$ is a partition in $(A, \cdot)$ such that $X \succ E$ for any partition $X$ in $(A, \cdot)$, and with the logical entropy $H_{l}^{\mu}(E)=0$. Evidently, $\bigvee_{k=0}^{n-1} U^{k}(E)=E$, hence $H_{l}^{\mu}(U, E)=0$.

Theorem 3.3 Let $(A, \mu, U)$ be a dynamical system in a product $M V$-algebra $(A, \cdot)$, and $X$ be a partition in $(A, \cdot)$. Then, for any non-negative integer $r$, the following equality holds:

$$
H_{l}^{\mu}(U, X)=H_{l}^{\mu}\left(U, \bigvee_{i=0}^{r} U^{i}(X)\right)
$$

Proof Using Definition 3.2, we can write

$$
\begin{aligned}
H_{l}^{\mu}\left(U, \bigvee_{i=0}^{r} U^{i}(X)\right) & =\lim _{n \rightarrow \infty} \frac{1}{n} H_{l}^{\mu}\left(\bigvee_{k=0}^{n-1} U^{k}\left(\bigvee_{i=0}^{r} U^{i}(X)\right)\right) \\
& =\lim _{n \rightarrow \infty} \frac{r+n}{n} \cdot \frac{1}{r+n} H_{l}^{\mu}\left(\bigvee_{k=0}^{r+n-1} U^{k}(X)\right) \\
& =\lim _{n \rightarrow \infty} \frac{1}{r+n} H_{l}^{\mu}\left(\bigvee_{k=0}^{r+n-1} U^{k}(X)\right)=H_{l}^{\mu}(U, X) .
\end{aligned}
$$

Theorem 3.4 Let $(A, \mu, U)$ be a dynamical system in a product $M V$-algebra $(A, \cdot)$, and $X, Y$ be partitions in $(A, \cdot)$ such that $Y \succ X$. Then $H_{l}^{\mu}(U, X) \leq H_{l}^{\mu}(U, Y)$.

Proof Let $Y \succ X$. By Propositions 2.3 and 3.1, we have $\bigvee_{k=0}^{n-1} U^{k}(Y) \succ \bigvee_{k=0}^{n-1} U^{k}(X)$ for $n=$ $1,2, \ldots$ Therefore, by property (L2), we get

$$
H_{l}^{\mu}\left(\bigvee_{k=0}^{n-1} U^{k}(X)\right) \leq H_{l}^{\mu}\left(\bigvee_{k=0}^{n-1} U^{k}(Y)\right)
$$

Consequently, dividing by $n$ and letting $n \rightarrow \infty$, we get $H_{l}^{\mu}(U, X) \leq H_{l}^{\mu}(U, Y)$. 
Definition 3.3 The logical entropy of a dynamical system $(A, \mu, U)$ in a product MValgebra $(A, \cdot)$ is defined by

$$
H_{l}^{\mu}(U)=\sup \left\{H_{l}^{\mu}(U, X) ; X \text { is a partition in }(A, \cdot)\right\} .
$$

Theorem 3.5 Let $(A, \mu, U)$ be a dynamical system in a product $M V$-algebra $(A, \cdot)$. Then, for every natural number $k$, it holds $H_{l}^{\mu}\left(U^{k}\right)=k \cdot H_{l}^{\mu}(U)$.

Proof Let $X$ be a partition in $(A, \cdot)$. Then, for every natural number $k$, we have

$$
\begin{aligned}
H_{l}^{\mu}\left(U^{k}, \bigvee_{j=0}^{k-1} U^{j}(X)\right) & =\lim _{n \rightarrow \infty} \frac{1}{n} H_{l}^{\mu}\left(\bigvee_{i=0}^{n-1}\left(U^{k}\right)^{i}\left(\bigvee_{j=0}^{k-1} U^{j}(X)\right)\right. \\
& =\lim _{n \rightarrow \infty} \frac{1}{n} H_{l}^{\mu}\left(\bigvee_{i=0}^{n-1} \bigvee_{j=0}^{k-1} U^{k+j}(X)\right)=\lim _{n \rightarrow \infty} \frac{1}{n} H_{l}^{\mu}\left(\bigvee_{j=0}^{n k-1} U^{j}(X)\right) \\
& =\lim _{n \rightarrow \infty} \frac{n k}{n} \frac{1}{n k} H_{l}^{\mu}\left(\bigvee_{j=0}^{n k-1} U^{j}(X)\right)=k \cdot H_{l}^{\mu}(U, X) .
\end{aligned}
$$

Hence we obtain

$$
\begin{aligned}
k \cdot H_{l}^{\mu}(U) & =k \cdot \sup \left\{H_{l}^{\mu}(U, X) ; X \text { is a partition in }(A, \cdot)\right\} \\
& =\sup \left\{H_{l}^{\mu}\left(U^{k}, \bigvee_{j=0}^{k-1} U^{j}(X)\right) ; X \text { is a partition in }(A, \cdot)\right\} \\
& \leq \sup \left\{H_{l}^{\mu}\left(U^{k}, Y\right) ; Y \text { is a partition in }(A, \cdot)\right\}=H_{l}^{\mu}\left(U^{k}\right) .
\end{aligned}
$$

On the other hand, by Proposition 2.2, we have $\bigvee_{j=0}^{k-1} U^{j}(X) \succ X$, and therefore, by Theorem 3.4, we get

$$
H_{l}^{\mu}\left(U^{k}, X\right) \leq H_{l}^{\mu}\left(U^{k}, \bigvee_{j=0}^{k-1} U^{j}(X)\right)=k \cdot H_{l}^{\mu}(U, X)
$$

It follows from this that

$$
\begin{aligned}
H_{l}^{\mu}\left(U^{k}\right) & =\sup \left\{H_{l}^{\mu}\left(U^{k}, X\right) ; X \text { is a partition in }(A, \cdot)\right\} \\
& \leq k \cdot \sup \left\{H_{l}^{\mu}(U, X) ; X \text { is a partition in }(A, \cdot)\right\}=k \cdot H_{l}^{\mu}(U) .
\end{aligned}
$$

In the rest of this section, we formulate a version of the Kolmogorov-Sinai theorem on generators for the case of the logical entropy of a dynamical system $(A, \mu, U)$.

Definition 3.4 Let $(A, \mu, U)$ be a dynamical system in a product MV-algebra $(A, \cdot)$. A partition $Z$ in $(A, \cdot)$ is said to be a generator of a dynamical system $(A, \mu, U)$ if to any partition $X$ in $(A, \cdot)$ there exists an integer $k>0$ such that $\bigvee_{i=0}^{k} U^{i}(Z) \succ X$.

Theorem 3.6 Let $Z$ be a generator of a dynamical system $(A, \mu, U)$. Then $H_{l}^{\mu}(U)=$ $H_{l}^{\mu}(U, Z)$. 
Proof Let $Z$ be a generator of a dynamical system $(A, \mu, U)$, and $X$ be any partition in $(A, \cdot)$. Then there exists an integer $k>0$ such that $\bigvee_{i=0}^{k} U^{i}(Z) \succ X$. Therefore, by Theorems 3.4 and 3.3, we have

$$
H_{l}^{\mu}(U, X) \leq H_{l}^{\mu}\left(U, \bigvee_{i=0}^{k} U^{i}(Z)\right)=H_{l}^{\mu}(U, Z),
$$

and consequently

$$
H_{l}^{\mu}(U)=\sup \left\{H_{l}^{\mu}(U, X) ; X \text { is a partition in }(A, \cdot)\right\}=H_{l}^{\mu}(U, Z) \text {. }
$$

\section{General type of entropy of dynamical systems in product MV-algebras}

In this section, we introduce, based on the function $\varphi:[0,1] \rightarrow \mathbb{R}$, a general type of entropy of a partition in a product MV-algebra $(A, \cdot)$ that contains the Shannon entropy and the logical entropy of a partition in a product $\mathrm{MV}$-algebra $(A, \cdot)$ as special cases. Subsequently, using the concept of $\varphi$-entropy of a partition in $(A, \cdot)$, where $\varphi$ is a so-called sub-additive generator [39], we define a general type of entropy of a dynamical system $(A, \mu, U)$, socalled $\varphi$-entropy of a dynamical system $(A, \mu, U)$. We construct for the proposed entropy measure an isomorphism theory of the Kolmogorov-Sinai type.

Definition 4.1 Let $X=\left(x_{1}, x_{2}, \ldots, x_{n}\right)$ be a partition in a product MV-algebra $(A, \cdot)$, and $\mu: A \rightarrow[0,1]$ be a state. If $\varphi:[0,1] \rightarrow \mathbb{R}$ is a function, then we define the $\varphi$-entropy of $X$ with respect to $\mu$ as the number

$$
H_{\varphi}^{\mu}(X)=\sum_{i=1}^{n} \varphi\left(\mu\left(x_{i}\right)\right)
$$

Example 4.1 If we put $\varphi=s$, where $s:[0,1] \rightarrow[0, \infty)$ is the Shannon entropy function defined by Eq. (1.1), then we obtain the Shannon entropy of $X$, and putting $\varphi=l$, where $l:[0,1] \rightarrow[0, \infty)$ is the logical entropy function defined by Eq. (1.2), the logical entropy of $X$ is obtained.

Definition 4.2 ([39]) A function $\varphi:[0,1] \rightarrow[0, \infty)$ is said to be a sub-additive generator if the following condition is satisfied: if $c_{i j} \in[0,1], i=1,2, \ldots, n, j=1,2, \ldots, m, \sum_{j=1}^{m} c_{i j}=a_{i}, i=$ $1,2, \ldots, n, \sum_{i=1}^{n} c_{i j}=b_{j}, j=1,2, \ldots, m$, and $\sum_{i=1}^{n} a_{i}=1, \sum_{j=1}^{m} b_{j}=1$, then

$$
\sum_{i=1}^{n} \sum_{j=1}^{m} \varphi\left(c_{i j}\right) \leq \sum_{i=1}^{n} \varphi\left(a_{i}\right)+\sum_{j=1}^{m} \varphi\left(b_{j}\right)
$$

Remark 4.1 In [39] we have shown that the Shannon entropy as well as the logical entropy functions are sub-additive generators. Moreover, a sub-additive generator different from these entropy functions was found; it was proven that the function $k:[0,1] \rightarrow[0, \infty)$ defined by

$$
k(x)=x\left(1-x^{2}\right)
$$

for every $x \in[0,1]$, is a sub-additive generator. The function $k$ will be called the quadratic logical entropy function. 
Remark 4.2 Consider any product MV-algebra $(A, \cdot)$ and the partition $E=(u)$ in $(A, \cdot)$. If $\varphi:[0,1] \rightarrow \mathbb{R}$ is a function with the property that $\varphi(1)=0$ (it is evident that all of the above three entropy functions satisfy this condition), then $H_{\varphi}^{\mu}(E)=0$.

Theorem 4.1 Let $\mu$ be a state on a product $M V$-algebra $(A, \cdot)$, and $\varphi$ be a sub-additive generator. Then, for any partitions $X, Y$ in a product $M V$-algebra $(A, \cdot)$, the following inequality holds:

$$
H_{\varphi}^{\mu}(X \vee Y) \leq H_{\varphi}^{\mu}(X)+H_{\varphi}^{\mu}(Y)
$$

Proof Suppose that $X=\left(x_{1}, x_{2}, \ldots, x_{n}\right)$ and $Y=\left(y_{1}, y_{2}, \ldots, y_{m}\right)$. Put $c_{i j}=\mu\left(x_{i} \cdot y_{j}\right), a_{i}=$ $\mu\left(x_{i}\right), b_{j}=\mu\left(y_{j}\right)$ for $i=1,2, \ldots, n, j=1,2, \ldots, m$. By Proposition 2.1, we get

$$
a_{i}=\mu\left(x_{i}\right)=\sum_{j=1}^{m} \mu\left(x_{i} \cdot y_{j}\right)=\sum_{j=1}^{m} c_{i j} \quad \text { and } \quad b_{j}=\mu\left(y_{j}\right)=\sum_{i=1}^{n} \mu\left(x_{i} \cdot y_{j}\right)=\sum_{i=1}^{n} c_{i j}
$$

for $i=1,2, \ldots, n, j=1,2, \ldots, m$. Further, according to Definition 2.2 and the definition of a partition in a product MV-algebra, we have

$$
\sum_{i=1}^{n} a_{i}=\sum_{i=1}^{n} \mu\left(x_{i}\right)=\mu\left(\sum_{i=1}^{n} x_{i}\right)=\mu(u)=1,
$$

analogously, we get that $\sum_{j=1}^{m} b_{j}=1$. Hence

$$
\begin{aligned}
H_{\varphi}^{\mu}(X \vee Y) & =\sum_{i=1}^{n} \sum_{j=1}^{m} \varphi\left(\mu\left(x_{i} \cdot y_{j}\right)\right)=\sum_{i=1}^{n} \sum_{j=1}^{m} \varphi\left(c_{i j}\right) \\
& \leq \sum_{i=1}^{n} \varphi\left(a_{i}\right)+\sum_{j=1}^{m} \varphi\left(b_{j}\right)=\sum_{i=1}^{n} \varphi\left(\mu\left(x_{i}\right)\right)+\sum_{j=1}^{m} \varphi\left(\mu\left(y_{j}\right)\right) \\
& =H_{\varphi}^{\mu}(X)+H_{\varphi}^{\mu}(Y) .
\end{aligned}
$$

To illustrate the result of the previous theorem, we provide the following example.

Example 4.2 Consider the measurable space $([0,1], B)$, where $B$ is the $\sigma$-algebra of all Borel subsets of the unit interval $[0,1]$. Let $A$ be a family of all Borel measurable functions $f:[0,1] \rightarrow[0,1]$. If we define in the family $A$ the operation- as the natural product of fuzzy sets, then the system $(A, \cdot)$ is a product MV-algebra. We define a state $\mu: A \rightarrow[0,1]$ by the equality $\mu(f)=\int_{0}^{1} f(x) d x$ for any element $f$ of $A$. It is easy to see that the pairs $X=\left(f_{1}, f_{2}\right), Y=\left(g_{1}, g_{2}\right)$, where $f_{1}(x)=x, f_{2}(x)=1-x, g_{1}(x)=x^{2}, g_{2}(x)=1-x^{2}, x \in[0,1]$, are two partitions in $(A, \cdot)$ with the state values $\frac{1}{2}, \frac{1}{2}$ and $\frac{1}{3}, \frac{2}{3}$ of the corresponding elements, respectively. The join of partitions $X$ and $Y$ is the system $X \vee Y=\left(f_{1} \cdot g_{1}, f_{1} \cdot g_{2}, f_{2} \cdot g_{1}, f_{2} \cdot g_{2}\right)$ with the state values $\frac{1}{4}, \frac{1}{4}, \frac{1}{12}, \frac{5}{12}$ of the corresponding elements. By simple calculations we get the Shannon entropies $H_{s}^{\mu}(X)=1, H_{s}^{\mu}(Y) \doteq 0.9183, H_{s}^{\mu}(X \vee Y) \doteq 1.8250$; the logical entropies $H_{l}^{\mu}(X)=0.5, H_{l}^{\mu}(Y) \doteq 0.4444, H_{l}^{\mu}(X \vee Y) \doteq 0.6944$; and the quadratic logical entropies $H_{k}^{\mu}(X)=0.75, H_{k}^{\mu}(Y) \doteq 0.6666, H_{k}^{\mu}(X \vee Y) \doteq 0.6615$. It is easy to see that for the subadditive generators $\varphi=s, \varphi=l$, and $\varphi=k$, it holds $H_{\varphi}^{\mu}(X \vee Y) \leq H_{\varphi}^{\mu}(X)+H_{\varphi}^{\mu}(Y)$, which is consistent with the claim of the previous theorem. 
Theorem 4.2 Let $(A, \mu, U)$ be a dynamical system in a product $M V$-algebra $(A, \cdot)$, and $\varphi:[0,1] \rightarrow \mathbb{R}$ be a function. Then, for any partition $X$ in $(A, \cdot)$ and for any non-negative integer $k$, it holds

$$
H_{\varphi}^{\mu}\left(U^{k}(X)\right)=H_{\varphi}^{\mu}(X)
$$

Proof The statement follows immediately from condition (iii) of Definition 3.1.

Proposition 4.1 Let $(A, \mu, U)$ be a dynamical system in a product $M V$-algebra $(A, \cdot)$, and $\varphi$ be a sub-additive generator. Then, for any partition $X$ in $(A, \cdot)$, there exists the following limit:

$$
\lim _{n \rightarrow \infty} \frac{1}{n} H_{\varphi}^{\mu}\left(\bigvee_{k=0}^{n-1} U^{k}(X)\right)
$$

Proof In view of sub-additivity of $\varphi$-entropy (Theorem 4.1) and the previous theorem, the proof can be made similarly as the proof of Proposition 3.2.

Definition 4.3 Let $(A, \mu, U)$ be a dynamical system in a product MV-algebra $(A, \cdot)$, and $\varphi$ be a sub-additive generator. Then we define the $\varphi$-entropy of $(A, \mu, U)$ by the formula

$$
H_{\varphi}^{\mu}(U)=\sup \left\{H_{\varphi}^{\mu}(U, X) ; X \text { is a partition in }(A, \cdot)\right\}
$$

where

$$
H_{\varphi}^{\mu}(U, X)=\lim _{n \rightarrow \infty} \frac{1}{n} H_{\varphi}^{\mu}\left(\bigvee_{k=0}^{n-1} U^{k}(X)\right)
$$

Example 4.3 It is clear that putting $\varphi=l$, where $l:[0,1] \rightarrow[0, \infty)$ is the logical entropy function defined by Eq. (1.2), we obtain the logical entropy of a dynamical system $(A, \mu, U)$. If we put $\varphi=s$, where $s:[0,1] \rightarrow[0, \infty)$ is the Shannon entropy function defined by Eq. (1.1), we obtain the Kolmogorov-Sinai entropy of a dynamical system $(A, \mu, U)$ defined and studied by Petrovičová in [37].

Definition 4.4 Two product MV-algebra dynamical systems $\left(A_{1}, \mu_{1}, U_{1}\right),\left(A_{2}, \mu_{2}, U_{2}\right)$ are said to be isomorphic if there exists some one-to-one and onto map $\psi: A_{1} \rightarrow A_{2}$ such that $\psi\left(u_{1}\right)=u_{2}$, and, for every $x, y \in A_{1}$, the following conditions are satisfied:

(i) $\psi(x \cdot y)=\psi(x) \cdot \psi(y)$;

(ii) if $x+y \leq u_{1}$, then $\psi(x+y)=\psi(x)+\psi(y)$;

(iii) $\mu_{2}(\psi(x))=\mu_{1}(x)$;

(iv) $\psi\left(U_{1}(x)\right)=U_{2}(\psi(x))$.

In this case, $\psi$ is called an isomorphism, and we write $U_{1} \cong U_{2}$.

Proposition 4.2 Let $\left(A_{1}, \mu_{1}, U_{1}\right),\left(A_{2}, \mu_{2}, U_{2}\right)$ be isomorphic product $M V$-algebra dynamical systems, and $\psi: A_{1} \rightarrow A_{2}$ be an isomorphism between $\left(A_{1}, \mu_{1}, U_{1}\right),\left(A_{2}, \mu_{2}, U_{2}\right)$. Then, for the inverse $\psi^{-1}: A_{2} \rightarrow A_{1}$, the following properties are satisfied:

(i) $\psi^{-1}(x \cdot y)=\psi^{-1}(x) \cdot \psi^{-1}(y)$ for every $x, y \in A_{2}$; 
(ii) if $x, y \in A_{2}$ such that $x+y \leq u_{2}$, then $\psi^{-1}(x+y)=\psi^{-1}(x)+\psi^{-1}(y)$;

(iii) $\mu_{1}\left(\psi^{-1}(x)\right)=\mu_{2}(x)$ for every $x \in A_{2}$;

(iv) $\psi^{-1}\left(U_{2}(x)\right)=U_{1}\left(\psi^{-1}(x)\right)$ for every $x \in A_{2}$.

Proof Since $\psi: A_{1} \rightarrow A_{2}$ is bijective, for every $x, y \in A_{2}$, there exist $x^{\prime}, y^{\prime} \in A_{1}$ such that $\psi^{-1}(x)=x^{\prime}$ and $\psi^{-1}(y)=y^{\prime}$.

(i) Let $x, y \in A_{2}$. Then we have

$$
\psi^{-1}(x \cdot y)=\psi^{-1}\left(\psi\left(x^{\prime}\right) \cdot \psi\left(y^{\prime}\right)\right)=\psi^{-1}\left(\psi\left(x^{\prime} \cdot y^{\prime}\right)\right)=x^{\prime} \cdot y^{\prime}=\psi^{-1}(x) \cdot \psi^{-1}(y) .
$$

(ii) Let $x, y \in A_{2}$ such that $x+y \leq u_{2}$. Then $x^{\prime}+y^{\prime} \leq u_{1}$, and

$$
\psi^{-1}(x+y)=\psi^{-1}\left(\psi\left(x^{\prime}\right)+\psi\left(y^{\prime}\right)\right)=\psi^{-1}\left(\psi\left(x^{\prime}+y^{\prime}\right)\right)=x^{\prime}+y^{\prime}=\psi^{-1}(x)+\psi^{-1}(y) .
$$

(iii) Let $x \in A_{2}$. Then $\mu_{2}(x)=\mu_{2}\left(\psi\left(x^{\prime}\right)\right)=\mu_{1}\left(x^{\prime}\right)=\mu_{1}\left(\psi^{-1}(x)\right)$.

(iv) Let $x \in A_{2}$. Then

$$
\psi^{-1}\left(U_{2}(x)\right)=\psi^{-1}\left(U_{2}\left(\psi\left(x^{\prime}\right)\right)\right)=\psi^{-1}\left(\psi\left(U_{1}\left(x^{\prime}\right)\right)\right)=U_{1}\left(x^{\prime}\right)=U_{1}\left(\psi^{-1}(x)\right) .
$$

Theorem 4.3 Let $\varphi$ be a sub-additive generator, and $\left(A_{1}, \mu_{1}, U_{1}\right),\left(A_{2}, \mu_{2}, U_{2}\right)$ be product $M V$-algebra dynamical systems. If $U_{1} \cong U_{2}$, then

$$
H_{\varphi}^{\mu_{1}}\left(U_{1}\right)=H_{\varphi}^{\mu_{2}}\left(U_{2}\right)
$$

Proof Let $\psi: A_{1} \rightarrow A_{2}$ be an isomorphism between dynamical systems $\left(A_{1}, \mu_{1}, U_{1}\right),\left(A_{2}\right.$, $\left.\mu_{2}, U_{2}\right)$. Consider a partition $X=\left(x_{1}, x_{2}, \ldots, x_{n}\right)$ in a product MV-algebra $\left(A_{1}, \cdot\right)$. Then $x_{1}+$ $x_{2}+\cdots+x_{n}=u_{1}$, and therefore, by condition (i) of Definition 4.4, it holds $\psi\left(x_{1}\right)+\psi\left(x_{2}\right)+$ $\cdots+\psi\left(x_{n}\right)=\psi\left(x_{1}+x_{2}+\cdots+x_{n}\right)=\psi\left(u_{1}\right)=u_{2}$. This means that the collection $\psi(X)=$ $\left(\psi\left(x_{1}\right), \psi\left(x_{2}\right), \ldots, \psi\left(x_{n}\right)\right)$ is a partition in a product MV-algebra $\left(A_{2}, \cdot\right)$. Moreover, according to condition (iii) of Definition 4.4, we have

$$
H_{\varphi}^{\mu_{2}}(\psi(X))=\sum_{i=1}^{n} \varphi\left(\mu_{2}\left(\psi\left(x_{i}\right)\right)\right)=\sum_{i=1}^{n} \varphi\left(\mu_{1}\left(x_{i}\right)\right)=H_{\varphi}^{\mu_{1}}(X)
$$

Hence, using conditions (iv) and (i) of Definition 4.4, we get

$$
\begin{aligned}
H_{\varphi}^{\mu_{2}}\left(\bigvee_{k=0}^{n-1} U_{2}^{k}(\psi(X))\right) & =H_{\varphi}^{\mu_{2}}\left(\bigvee_{k=0}^{n-1} \psi\left(U_{1}^{k}(X)\right)\right) \\
& =H_{\varphi}^{\mu_{2}}\left(\psi\left(\bigvee_{k=0}^{n-1} U_{1}^{k}(X)\right)\right)=H_{\varphi}^{\mu_{1}}\left(\bigvee_{k=0}^{n-1} U_{1}^{k}(X)\right)
\end{aligned}
$$

Therefore, dividing by $n$ and letting $n \rightarrow \infty$, we obtain

$$
H_{\varphi}^{\mu_{2}}\left(U_{2}, \psi(X)\right)=\lim _{n \rightarrow \infty} \frac{1}{n} H_{\varphi}^{\mu_{2}}\left(\bigvee_{k=0}^{n-1} U_{2}^{k}(\psi(X))\right)
$$




$$
=\lim _{n \rightarrow \infty} \frac{1}{n} H_{\varphi}^{\mu_{1}}\left(\bigvee_{k=0}^{n-1} U_{1}^{k}(X)\right)=H_{\varphi}^{\mu_{1}}\left(U_{1}, X\right) .
$$

This implies that

$$
\left\{H_{\varphi}^{\mu_{1}}\left(U_{1}, X\right) ; X \text { is a partition in }\left(A_{1}, \cdot\right)\right\} \subset\left\{H_{\varphi}^{\mu_{2}}\left(U_{2}, Y\right) ; Y \text { is a partition in }\left(A_{2}, \cdot\right)\right\} \text {, }
$$

and consequently

$$
\begin{aligned}
H_{\varphi}^{\mu_{1}}\left(U_{1}\right) & =\sup \left\{H_{\varphi}^{\mu_{1}}\left(U_{1}, X\right) ; X \text { is a partition in }\left(A_{1}, \cdot\right)\right\} \\
& \leq \sup \left\{H_{\varphi}^{\mu_{2}}\left(U_{2}, Y\right) ; Y \text { is a partition in }\left(A_{2}, \cdot\right)\right\}=H_{\varphi}^{\mu_{2}}\left(U_{2}\right)
\end{aligned}
$$

The converse $H_{\varphi}^{\mu_{2}}\left(U_{2}\right) \leq H_{\varphi}^{\mu_{1}}\left(U_{1}\right)$ is obtained in a similar way; according to Proposition 4.2, it suffices to consider the inverse $\psi^{-1}: A_{2} \rightarrow A_{1}$.

By combining the previous results, we obtain the following statement.

Corollary 4.1 If $U_{1} \cong U_{2}$, then

(i) $H_{s}^{\mu_{1}}\left(U_{1}\right)=H_{s}^{\mu_{2}}\left(U_{2}\right)$;

(ii) $H_{l}^{\mu_{1}}\left(U_{1}\right)=H_{l}^{\mu_{2}}\left(U_{2}\right)$;

(iii) $H_{k}^{\mu_{1}}\left(U_{1}\right)=H_{k}^{\mu_{2}}\left(U_{2}\right)$.

Remark 4.3 It trivially follows from the above theorem that if $H_{\varphi}^{\mu_{1}}\left(U_{1}\right) \neq H_{\varphi}^{\mu_{2}}\left(U_{2}\right)$, then the corresponding dynamical systems $\left(A_{1}, \mu_{1}, U_{1}\right),\left(A_{2}, \mu_{2}, U_{2}\right)$ are not isomorphic. This means that the proposed $\varphi$-entropy distinguishes non-isomorphic product $M V$-algebra dynamical systems.

\section{Conclusions}

In the paper we have extended the results concerning the logical entropy of partitions in product MV-algebras provided in [1] to the case of dynamical systems. By using the concept of logical entropy of a partition in a product MV-algebra, we introduced the notion of logical entropy of a product MV-algebra dynamical system and derived the basic properties of this measure of information. In particular, a logical version of the Kolmogorov-Sinai theorem on generators was provided.

In addition, using the concept of the sub-additive generator $\varphi$ introduced by the authors in [39], we have defined a general type of entropy of a product MV-algebra dynamical system $(A, \mu, U)$, the so-called $\varphi$-entropy of a dynamical system $(A, \mu, U)$. The proposed $\varphi$ entropy includes the logical entropy and the Kolmogorov-Sinai entropy as special cases: if we put $\varphi=l$, where $l:[0,1] \rightarrow[0, \infty)$ is the logical entropy function defined by Eq. (1.2), we obtain the logical entropy of a dynamical system $(A, \mu, U)$, and putting $\varphi=s$, where $s:[0,1] \rightarrow[0, \infty)$ is the Shannon entropy function defined by Eq. (1.1), we obtain the Kolmogorov-Sinai entropy of a dynamical system $(A, \mu, U)$ defined and studied by Petrovičová in [37]. For the proposed $\varphi$-entropy $H_{\varphi}^{\mu}(U)$, we have created an isomorphism theory of the Kolmogorov-Sinai type. It was shown that the $\varphi$-entropy $H_{\varphi}^{\mu}(U)$ distinguishes nonisomorphic product MV-algebra dynamical systems. In this way, we have acquired several instruments to distinguish non-isomorphic product MV-algebra dynamical systems: the 
logical, the Kolmogorov-Sinai, and the quadratic logical entropy of a dynamical system $(A, \mu, U)$.

As mentioned above (see Example 3.2), the full tribe of fuzzy sets represents a special case of product MV-algebras; the obtained results can therefore be immediately applied to this significant family of fuzzy sets. From the point of view of applications, it is interesting that to a given family $\mathcal{F}$ of intuitionistic fuzzy sets can be constructed an MV-algebra $\mathcal{A}$ such that $\mathcal{F}$ can be embedded to $\mathcal{A}$. Also, product on $\mathcal{F}$ can be introduced by such a way that the corresponding MV-algebra is an MV-algebra with product. Hence all results of our paper can be applied also to the case of intuitionistic fuzzy sets.

\section{Acknowledgements}

The authors would like to thank the editors and the reviewers for their valuable comments and constructive suggestions that have improved the quality and presentation of this paper.

\section{Funding}

Not applicable.

Availability of data and materials

Not applicable.

\section{Competing interests}

The authors declare that they have no competing of interest.

\section{Authors' contributions}

Both authors have contributed significantly and equally in writing this article. Both authors read and approved the final manuscript.

\section{Author details}

${ }^{1}$ Department of Mathematics, Faculty of Natural Sciences, Constantine the Philosopher University in Nitra, Nitra, Slovakia. ${ }^{2}$ Department of Mathematics, Faculty of Natural Sciences, Matej Bel University, Banská Bystrica, Slovakia. ${ }^{3}$ Mathematical Institute, Slovak Academy of Sciences, Bratislava, Slovakia.

\section{Publisher's Note}

Springer Nature remains neutral with regard to jurisdictional claims in published maps and institutional affiliations.

Received: 20 June 2018 Accepted: 2 January 2019 Published online: 14 January 2019

\section{References}

1. Markechová, D., Mosapour, B., Ebrahimzadeh, A.: Logical divergence, logical entropy, and logical mutual information in product MV-algebras. Entropy 20, Article ID 129 (2018). https://doi.org/10.3390/e20020129

2. Shannon, C.E.: A mathematical theory of communication. Bell Syst. Tech. J. 27, 379-423 (1948)

3. Gray, R.M.: Entropy and Information Theory. Springer, Berlin (2009)

4. Kolmogorov, A.N.: New metric invariant of transitive dynamical systems and automorphisms of Lebesgue spaces. Dokl. Russ. Acad. Sci. 119, 861-864 (1958)

5. Sinai, Y.G.: On the notion of entropy of a dynamical system. Dokl. Russ. Acad. Sci. 124, 768-771 (1959)

6. Ornstein, D.: Bernoulli shifts with the same entropy are isomorphic. Adv. Math. 4, 337-352 (1970)

7. Ornstein, D.: Two Bernoulli shifts with infinite entropy are isomorphic. Adv. Math. 5, 333-458 (1971)

8. Markechová, D., Ebrahimzadeh, A., Eslami Giski, Z.: Logical entropy of dynamical systems. Adv. Differ. Equ. 2018, Article ID 70 (2018). https://doi.org/10.1186/s13662-018-1524-z

9. Ellerman, D.: Logical information theory: new foundations for information theory. Log. J. IGPL 25, $806-835$ (2017)

10. Markechová, D., Riečan, B.: Logical entropy of fuzzy dynamical systems. Entropy 18, Article ID 157 (2016). https://doi.org/10.3390/e18040157

11. Mohammadi, U.: The concept of logical entropy on D-posets. J. Algebraic Struct. Appl. 1, 53-61 (2016)

12. Ebrahimzadeh, A.: Logical entropy of quantum dynamical systems. Open Phys. 14, 1-5 (2016)

13. Ebrahimzadeh, A.: Quantum conditional logical entropy of dynamical systems. Ital. J. Pure Appl. Math. 36, 879-886 (2016)

14. Markechová, D., Riečan, B.: Logical entropy and logical mutual information of experiments in the intuitionistic fuzzy case. Entropy 19, Article ID 429 (2017). https://doi.org/10.3390/e190804

15. Ebrahimzadeh, A., Eslami Giski, Z., Markechová, D.: Logical entropy of dynamical systems—a general model. Mathematics 5, Article ID 4 (2017). https://doi.org/10.3390/math5010004

16. Ebrahimzadeh, A., Jamalzadeh, J.: Conditional logical entropy of fuzzy $\sigma$-algebras. J. Intell. Fuzzy Syst. 33, 1019-1026 (2017)

17. Eslami Giski, Z., Ebrahimzadeh, A.: An introduction of logical entropy on sequential effect algebra. Indag. Math. 28, 928-937 (2017)

18. Zadeh, L.A.: Fuzzy sets. Inf. Control 8, 338-358 (1965) 
19. Zadeh, L.A.: Probability measures of fuzzy events. J. Math. Anal. Appl. 23, 421-427 (1968)

20. Markechová, D.: The entropy of fuzzy dynamical systems and generators. Fuzzy Sets Syst. 48, 351-363 (1992)

21. Dumitrescu, D.: Entropy of a fuzzy dynamical system. Fuzzy Sets Syst. 70, 45-57 (1995)

22. Riečan, B., Markechová, D.: The entropy of fuzzy dynamical systems, general scheme, and generators. Fuzzy Sets Syst. 96, 191-199 (1998)

23. Markechová, D., Riečan, B.: Entropy of fuzzy partitions and entropy of fuzzy dynamical systems. Entropy 18, Article ID 19 (2016). https://doi.org/10.3390/e18010019

24. Riečan, B., Neubrunn, T.: Integral, Measure and Ordering. Kluwer, Dordrecht (1997)

25. Atanassov, K.: Intuitionistic fuzzy sets. Fuzzy Sets Syst. 20, 87-96 (1986)

26. Riečan, B.: Probability theory on IF events. In: Algebraic and Proof-Theoretic Aspects of Non-Classical Logics; Papers in Honor of Daniele Mundici on the Occasion of His 60th Birthday. Lecture Notes in Computer Science, pp. 290-308. Springer, New York (2007)

27. Chang, C.C.: Algebraic analysis of many valued logics. Trans. Am. Math. Soc. 88, 467-490 (1958)

28. Mundici, D.: Interpretation of AFC*-algebras in Łukasiewicz sentential calculus. J. Funct. Anal. 56, 889-894 (1986)

29. Di Nola, A., Dvurečenskij, A., Hyčko, M., Manara, C.: Entropy on effect algebras with the Riesz decomposition property II: MV-algebras. Kybernetika 41, 161-176 (2005)

30. Riečan, B.: Kolmogorov-Sinaj entropy on MV-algebras. Int. J. Theor. Phys. 44, 1041-1052 (2005)

31. Riečan, B., Mundici, D.: Probability on MV-algebras. In: Pap, E. (ed.) Handbook of Measure Theory, pp. 869-910. Elsevier, Amsterdam (2002)

32. Riečan, B.: On the product MV-algebras. Tatra Mt. Math. Publ. 16, 143-149 (1999)

33. Montagna, F.: An algebraic approach to propositional fuzzy logic. J. Log. Lang. Inf. 9(1, 91-124 (2000). Special issue on Logics of Uncertainty

34. Jakubík, J.: On product MV algebras. Czechoslov. Math. J. 52, 797-810 (2002)

35. Di Nola, A., Dvurečenskij, A.: Product MV-algebras. Mult. Valued Log. 6, 193-215 (2001)

36. Petrovičová, J.: On the entropy of partitions in product MV-algebras. Soft Comput. 4, 41-44 (2000)

37. Petrovičová, J.: On the entropy of dynamical systems in product MV-algebras. Fuzzy Sets Syst. 121, 347-351 (2001)

38. Markechová, D., Riečan, B.: Kullback-Leibler divergence and mutual information of partitions in product MV algebras. Entropy 19, Article ID 267 (2017). https://doi.org/10.3390/e19060267

39. Riečan, B., Markechová, D.: $\varphi$-Entropy of IF-partitions. Notes IFS 23, 9-15 (2017)

40. Gluschankof, D.: Cyclic ordered groups and MV-algebras. Czechoslov. Math. J. 43, 249-263 (1993)

41. Cattaneo, G., Lombardo, F.: Independent axiomatization for MV-algebras. Tatra Mt. Math. Publ. 15, 227-232 (1998)

42. Riečan, B.: Analysis of fuzzy logic models. In: Koleshko, V.M. (ed.) Intelligent Systems. InTech Janeza Trdine 9, Rijeka, pp. 219-244 (2012)

43. Mundici, D.: Advanced Łukasiewicz Calculus and MV-Algebras. Springer, Dordrecht (2011)

44. Anderson, M., Feil, T.: Lattice Ordered Groups. Kluwer, Dordrecht (1988)

45. Riečan, B.: On the probability theory on product MV-algebras. Soft Comput. 4, 49-57 (2000)

46. Kroupa, T.: Conditional probability on MV-algebras. Fuzzy Sets Syst. 149, 369-381 (2005)

47. Vrábelová, M.: A note on the conditional probability on product MV algebras. Soft Comput. 4, 58-61 (2000)

48. Walters, P: An Introduction to Ergodic Theory. Springer, New York (1982)

\section{Submit your manuscript to a SpringerOpen ${ }^{\circ}$ journal and benefit from:}

- Convenient online submission

- Rigorous peer review

- Open access: articles freely available online

- High visibility within the field

- Retaining the copyright to your article

Submit your next manuscript at $\gg$ springeropen.com 